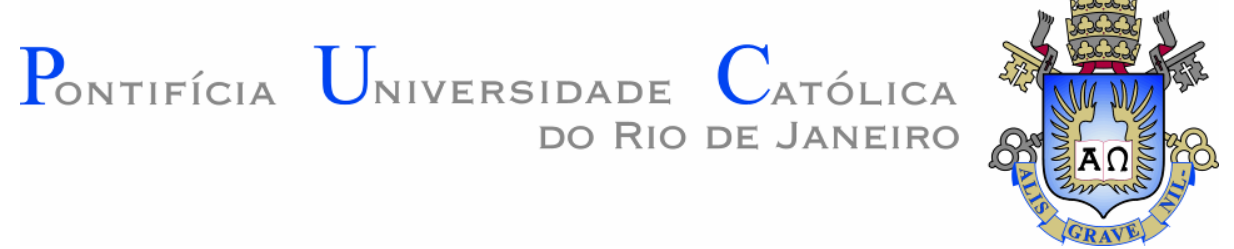

Wilson Silva Prata

\title{
Usabilidade nas Lojas de Aplicativos para Smartphones
}

Dissertação de Mestrado

Dissertação apresentada como requisito parcial para obtenção do título de Mestre pelo Programa de PósGraduação em Design da PUC-Rio.

Orientadoras: Prof. ${ }^{a}$ Claúdia Mont'Alvão Prof. ${ }^{a}$ Anamaria de Moraes Co-orientadora: Prof. ${ }^{a}$ Manuela Quaresma 


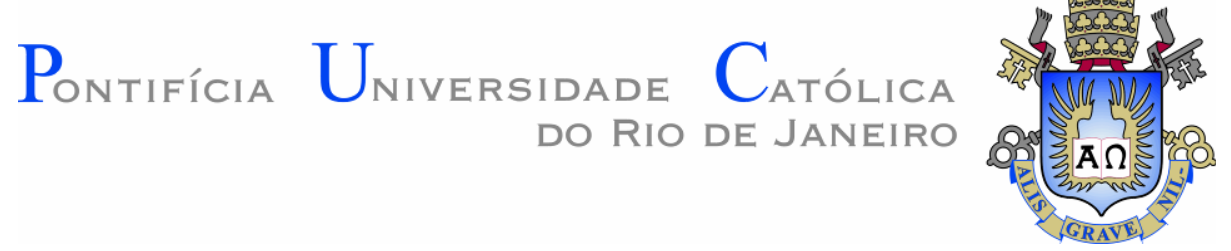

Wilson Silva Prata

\title{
Usabilidade nas Lojas de Aplicativos \\ para Smartphones
}

\begin{abstract}
Dissertação apresentada como requisito parcial para obtenção do título de Mestre pelo Programa de PósGraduação em Design do Departamento de Artes \& Design da PUC-Rio. Aprovada pela Comissão Examinadora abaixo assinada.
\end{abstract}

Prof.a Cláudia Renata Mont'Alvão Bastos Rodrigues Orientadora - Departamento de Artes \& Design - PUC-Rio

Prof.a Maria Manuela Rupp Quaresma Co-orientadora - Departamento de Artes \& Design - PUC-Rio

Prof.a Luiza Novaes Departamento de Artes \& Design - PUC-Rio

Prof. Eduardo Ariel de Souza Texeira Escola Superior de Propaganda e Marketing - ESPM

Prof. Leonardo Marques de Abreu Escola Superior de Propaganda e Marketing - ESPM

Prof.a Denise Berruezo Portinari Coordenadora Setorial do Centro de Teologia e Ciências Humanas - PUC-Rio

Rio de Janeiro, 27 de fevereiro de 2013. 
Todos os direitos reservados. É proibida a reprodução total ou parcial do trabalho sem autorização da universidade, da autora e do orientador.

\section{Wilson Silva Prata}

Graduado em Desenho Industrial na Universidade Federal do Amazonas, possui especialização em Tecnologia Multimídia pela mesma instituição e MBA em Marketing pela FVG/ISAE-AM.

Ficha Catalográfica

Prata, Wilson Silva

Usabilidade nas lojas de aplicativos para smartphones / Wilson Silva Prata ; orientadores: Claúdia Mont'Alvão, Anamaria de Moraes ; co-orientadora: Manuela Quaresma. - 2013.

209 f. : il. (color) ; $30 \mathrm{~cm}$

Dissertação (mestrado)-Pontifícia Universidade Católica do Rio de Janeiro, Departamento de Artes e Design, 2013.

Inclui bibliografia

1. Artes e design - Teses. 2. Ergonomia. 3. Usabilidade. 4. Interação humano-computador. 5. Lojas de aplicativos. 6. Dispositivos móveis. 7. Smartphones. I. Mont'Alvão, Claúdia. II. Moraes, Anamaria de. III. Quaresma, Manuela. IV. Pontifícia Universidade Católica do Rio de Janeiro. Departamento de Artes \& Design. V. Título. 


\section{Agradecimentos}

À professora Anamaria de Moraes, in memoriam, por ter acreditado no potencial desse trabalho e por todo apoio e recomendações.

À Professora Manuela Quaresma, por ter acompanhado todo essa jornada, mesmo aos domingos, mesmo à distância.

À Professora Cláudia Mont'Alvão por ter aceito a orientação em um momento crítico e ter conduzido e orientado a pesquisa.

À Fran, por ser meu porto seguro e estar sempre ao meu lado.

À minha filha Luane Maria e a minha família.

Aos funcionários e professores do Departamento de Artes \& Design da PUC-Rio.

Aos respondentes do questionário e participantes do teste de usabilidade, sem eles seria impossível esse trabalho.

À coordenação de Aperfeiçoamento de Pessoal de Nível Superior (CAPES) e a Fundação de Amparo à Pesquisa do Estado do Rio de Janeiro (FAPERJ), pelo suporte financeiro recebido ao longo do curso. 


\section{Resumo}

Prata, Wilson Silva; Moraes, Anamaria; Mont'Alvão, Cláudia; Quaresma, Manuela. Usabilidade nas Lojas de Aplicativos para Smartphones. Rio de Janeiro, 2013. 209p. Dissertação de Mestrado Departamento de Artes \& Design, Pontifícia Universidade do Rio de Janeiro.

Lojas de aplicativos são soluções desenvolvidas para distribuição de conteúdo digital para dispositivos móveis. As lojas estão presentes em quase todos os smartphones comercializados no país e possuem forte apelo frente ao público e aos desenvolvedores locais. Entretanto, por ser um canal relativamente novo, com pouco mais de seis anos de uso e popularização, ainda não há uma bibliografia fortemente estabelecida a esse respeito no que tange à Usabilidade e Interação Humano-Computador. Essas referências ficam ainda mais rarefeitas quando se procura por fontes que tratem da realidade brasileira levando em conta suas particularidades. O presente trabalho buscou colaborar para a formação dessa bibliografia através de uma pesquisa cujo o foco foram as principais lojas de aplicativos para smartphones disponíveis no país. Para isso, foi analisado o desenvolvimento histórico da comunicação móvel no Brasil e no mundo, para que se pudesse entender quais necessidades sociais possibilitaram o surgimento dos smartphones e legitimaram as lojas como solução de distribuição de conteúdo para esses aparelhos. Após isso, uma pesquisa de campo foi feita para identificação do perfil do usuário brasileiro. Para essa etapa, usou-se um questionário online. Com a análise dos dados foi possível determinar o perfil geral desses usuários. Essas informações foram a base para o desenvolvimento do perfil e seleção dos participantes da segunda técnica de pesquisa utilizada, um Teste de Usabilidade. Nesse teste, foram avaliadas as tarefas de busca, aquisição e avaliação nas duas principais lojas de aplicativos. Com esses achados foi possível confirmar e identificar vários problemas nas lojas. Alguns desses são os mesmos reportados por outras pesquisa realizadas no exterior, mostrando que vários problemas apresentados em outros locais também se repetem aqui 
no Brasil. Contudo, os dados mais relevantes foram aqueles particulares realidade nacional, como a importância atribuída as comunidades digitais, preferências no uso da loja e problemas relacionados a terminologias utilizadas nas lojas. Ao final do trabalho uma lista de parâmetros e recomendações para lojas de aplicativos foi desenvolvida com base nos achados das técnicas de pesquisa utilizadas.

\section{Palavras-chave}

Ergonomia; Usabilidade; Interação Humano-Computador; Lojas de aplicativos; Dispositivos móveis; Smartphones. 


\section{Abstract}

Prata, Wilson Silva; Moraes, Anamaria (Advisor); Mont'Alvão, Cláudia (Advisor); Quaresma, Manuela (Co-advisor). Usability in smartphone app store. Rio de Janeiro, 2013. 209p. MSc. Dissertation Departamento de Artes \& Design, Pontifícia Universidade do Rio de Janeiro.

Mobile Applications Stores are content distribution solution dedicated to mobile devices. These solutions are presented in most of the Smartphone available in Brazil and - as a content distribution solution - it has a strong appeal to developers and users. However, since it is a new model of distribution, there is not yet a core bibliography established concerning Usability and HumanComputer Interaction. There are even fewer references when the focus is the Brazilian market and its particularities. This research aimed to fulfill this gap through a theoretical and practical study of the major mobile application's stores available in Brazil. It started with a historical investigation of the development of mobile communication technologies in Brazil and in the world. The purpose of this investigation was to understand the social needs that make it possible the development of Smartphone and mobile application's stores; and how its became the default solution to mobile content distribution, nowadays. After that, a field research was conducted to identify the profile of the Brazilian mobiles application's stores user. A questionnaire was chosen as research's method. The data analysis of the questionnaire's entries enables the identification of this profile; it was used as reference to gather users to an Usability Test. The Test evaluated the methods of search, acquisition and evaluation in the two biggest stores available in Brazil. With these findings it was possible to confirm and identify many of the problems of the stores. Some of these problems are the same reported by other research. It demonstrates that various interactions' problems presented elsewhere are present here in Brazil as well. However, the most relevant data concern to Brazilian audience particularity, e.g. the relevance attributed to digital communities, the preferences in store usage and 
problems regarding the use of unknown terms and labels. At the end of this document there is a list of parameters and recommendations that was developed with the finding of both research techniques.

\section{Keywords}

Ergonomics; Usability; Human-Computer Interaction; Mobile Application's Store; Mobile Devices; Smartphone. 


\section{Sumário}

1. INTRODUÇÃO 16

2. DOS TELEGRÁFOS À TELEFONIA MÓVEL 23

2.1. Tecnologias de telefonia móvel 24

2.2. A Telefonia no Brasil 26

2.3. Celulares, feature phones e smartphones $\quad 27$

2.4. Dispositivos móveis no Brasil 28

2.5. Operadoras de telefonia móvel no Brasil 32

2.6. Tempo, espaço e comunicação na modernidade 33

3. DISTRIBUIÇÃO DE CONTEÚDO PARA DISPOSITIVOS MÓVEIS: AS LOJAS DE APLICATIVOS 38

3.1. Lojas de aplicativos para dispositivos móveis $\quad 42$

3.1.1. iPhone App Store $\quad 43$

3.1.2. Google Play Store 44

3.1.3. Nokia Ovi Store 45

3.1.4. BlackBerry App World 45

3.2. Interface das Lojas de Aplicativos $\quad 45$

3.3. Sistemas de Organização 46

3.3.1. iPhone App Store $\quad 47$

3.3.2. Google Play Store $\quad 48$

3.3.3. Loja Nokia $\quad 50$

3.3.4. BlackBerry 51

3.4. Sistemas de Rotulação 52

3.4.1. iPhone App Store 53

3.4.2 Google Play Store $\quad 53$

3.4.3. Loja Nokia 54

3.4.4. BlackBerry App World 54

3.5. Sistemas de Navegação $\quad 54$

3.5.1. iPhone App Store $\quad 55$

3.5.2. Google Play Store $\quad 55$

3.5.3. Nokia Ovi Store $\quad 56$ 
3.5.4. BlackBerry App World 56

3.6. Sistemas de busca 56

3.6.1. iPhone App Store $\quad 59$

3.6.2. Google Play Store $\quad 60$

3.6.3. Loja Nokia $\quad 60$

3.6.4. BlackBerry App World 61

3.7. Avaliação geral das Lojas de aplicativos 61

4. USABILIDADE E DISPOSITIVOS MÓVEIS 63

4.1. Usabilidade e Dispositivos móveis 68

4.2. Usabilidade e Experiência do Usuário (UX) 73

4.3. Usabilidade e Experiência do Usuário em dispositivos móveis 80

$\begin{array}{ll}\text { 4.4. Conclusão do capítulo } & 87\end{array}$

5. MÉTODOS E TÉCNICAS DE PESQUISA 91

5.1. Delineamento da Pesquisa 91

$\begin{array}{ll}\text { 5.1.1. Tema } & 91\end{array}$

5.1.2. Problema $\quad 91$

5.1.3. Objeto da Pesquisa $\quad 92$

5.1.4. Hipóteses e Variáveis $\quad 92$

5.1.5. Objetivos 92

5.1.6. Justificativa e aplicabilidade 93

5.2. Método, Técnicas e Procedimentos 94

5.2.1. Questionário 94

5.2.1.1. Propostas e Objetivos 95

5.2.1.2. Questões da Pesquisa 96

5.2.1.3. Estrutura do Questionário 96

5.2.1.4. Teste Piloto 98

5.2.1.5. Formato, Aplicação e Promoção 99

5.2.1.6. Análise dos dados 100

$\begin{array}{ll}\text { 5.2.2.Teste de Usabilidade } & 100\end{array}$

5.2.2.1. Propostas e Objetivos 100

5.2.2.2. Questões da Pesquisa 101

5.2.2.3. Tarefas e Cenários 102

5.2.2.4. Perfil dos Participantes 103

$\begin{array}{ll}\text { 5.2.2.5. Sistemas Avaliados } & 104\end{array}$ 
5.2.2.6. Ambiente e Equipamento do Teste 104

5.2.2.7. Procedimentos das Sessões do Teste 105

5.2.2.8. Dados Coletados 108

5.2.2.9. Teste Piloto 109

5.3. Conclusão do capítulo 110

6. ANÁLISE DOS RESULTADOS DA TÉCNICA DE QUESTIONÁRIO 113

6.1. Perfil dos Respondentes 113

6.2. Análise dos Resultados das Medidas Coletadas 115

6.2.1. Uso das lojas e dos aplicativos no Brasil $\quad 117$

6.2.2. Preferências no uso das lojas de aplicativos $\quad 120$

6.2.3. Satisfação nas lojas de aplicativos $\quad 125$

6.2.4. Comentários dos usuários $\quad 129$

6.3. Síntese dos resultados 134

7. ANÁliSE dOS RESULTADOS DO TESTE DE USABILIDADE 135

7.1. Perfil dos Respondentes 135

7.2. Completude das tarefas nos sistemas 137

7.2.1. Tarefa 1 - Baixar o aplicativo Angry Birds 144

7.2.2. Tarefa 2 - Comentar o aplicativo Angry Birds 146

7.2.3. Tarefa 3 - Baixar um aplicativo gratuito da área de destaque 148

7.2.4. Tarefa 4 - Baixar uma aplicativo de clima 150

7.2.5. Tarefa 5 - Baixar um navegador 151

7.3. Satisfação nas lojas de aplicativos 153

7.4. Comentários dos participantes em relação as lojas 156

7.4.1. Comentários em relação ao processo de entrada de dados 156

7.4.2. Comentários em relação a quantidade e qualidade das informações 158

7.4.3. Comentários em relação a ferramenta de busca 159

7.4.4. Comentários em relação a organização da loja 161

7.4.5. Comentários Gerais em relação às lojas 162

7.5. Síntese dos resultados 164

8. CONCLUSÃO 166

8.1. Desdobramentos da pesquisa 170

$\begin{array}{ll}\text { 8.2. Lições aprendidas } & 170\end{array}$ 
9. RECOMENDAÇÕES DE USABILIDADE PARA LOJAS DE APLICATIVOS PARA DISPOSITIVOS MÓVEIS

9.1. Comunicação e organização 172

9.2. Entrada de dados 174

9.3. Busca 175

9.4. Avaliação 176

REFERÊNCIAS BIBLIOGRÁFICAS 178

Apêndice I - Questionário 186

$\begin{array}{ll}\text { Apêndice II - Teste de Usabilidade } & 197\end{array}$ 


\section{Lista de figuras}

Figura 3.1: Lojas de aplicativos para dispositivos móveis $\quad 40$

$\begin{array}{ll}\text { Figura 3.2: Ícone das lojas de Aplicativos } & 47\end{array}$

Figura 3.3: iPhone App Store 48

Figura 3.4: Google Play Store $\quad 49$

Figura 3.5: Loja Nokia $\quad 50$

Figura 3.6: Loja BlackBerry App World 52

Figura 4.1: Contexto de interação em dispositivos móveis $\quad 77$

Figura 4.2: Mapa da Experiência do Usuário 89

Figura 4.3: Câmera de documentos $\quad 85$

Figura 4.4: Câmera acoplada ao celular 86

Figura 5.1: Estrutura do Questionário $\quad 97$

Figura 5.2: Samsung Galaxy Ace e iPhone 3GS 104 


\section{Lista de Gráficos}

Gráfico 6.1: Idade dos respondentes 113

Gráfico 6.2: Sexo dos respondentes 114

Gráfico 6.3 e 6.4: Tempo de uso de smartphones e tempo de uso do smartphone atual

Gráfico 6.5: Nível de escolaridade 114

Gráfico 6.6: Funções mais utilizadas 116

Gráfico 6.7: Freqüência de uso das lojas de aplicativos 117

Gráfico 6.8: Freqüência de uso dos aplicativos instalados 118

Gráfico 6.9: Quantidade de aplicativos instalados 118

Gráfico 6.10: Quantidade de aplicativos baixados por semana 119

Gráfico 6.11: Comparativo entre a preferência entre

baixar aplicativos no celular, no computador ou em ambos 120

Gráfico 6.12: Ocorrência de desistência na compra de um aplicativo 122

Gráfico 6.13: Freqüência de ocorrência de busca por um aplicativo sem resultados satisfatórios 124

Gráfico 6.14: Ocorrência da atividade de comentário e avaliação nas lojas 125

Gráfico 6.15: Ocorrência da atividade de comentário e avaliação nas lojas 126

Gráfico 6.16: O que o usuário faz com um aplicativo que o tenha decepcionado

Gráfico 6.17: Ocorrência da atividade de comentário e avaliação nas lojas 128

Gráfico 6.18: Ocorrência da atividade de comentário e avaliação nas lojas 128

Gráfico 7.1: Idade dos respondentes 135

Gráfico 7.2: Sexo dos respondentes 136

Gráfico 7.3: Experiência de uso das lojas de aplicativos 136

$\begin{array}{ll}\text { Gráfico 7.4: Tempo de uso de smartphones } & 137\end{array}$

Gráfico 7.5: Nível de escolaridade $\quad 137$

Gráfico 7.6: Completude das tarefas nos sistemas testados 139

Gráfico 7.7: Nível de completude das tarefas nos sistemas testados $\quad 140$

Gráfico 7.8: Completude das tarefas em usuários

com experiência no uso das lojas de aplicativos

Gráfico 7.9: Completude das tarefas em usuários sem experiência no uso das lojas de aplicativos 
Gráfico 7.10: Completude das tarefas com base na

experiência do participante

Gráfico 7.11: Completude das tarefas 142

Gráfico 7.12: Completude das tarefas na loja Android 143

Gráfico 7.13: Completude das tarefas na loja iPhone 143

Gráfico 7.14: Completude da tarefa 1

Gráfico 7.15: Completude da tarefa $2 \quad 146$

Gráfico 7.16: Completude da tarefa 3 149

Gráfico 7.17: Completude da tarefa 4

Gráfico 7.18: Completude da tarefa 5

Gráfico 7.19: Resultado do Questionário SUS 154

Gráfico 7.20: Distribuição da freqüência de resultados do Questionário SUS

\section{Lista de tabelas}

Tabela 1.1: Relação dos capítulos da dissertação, seus objetivos e conteúdo 20

Tabela 2.1: Cobertura da Tecnologia 3G (WCDMA) por

$\begin{array}{ll}\text { Região SMC em Jul/12 } & 30\end{array}$

Tabela 3.1: Cinco principais fabricantes mundiais de Smartphones 41

Tabela 7.1: Tarefas, objetivos e critérios para a completude 138 